\title{
Evaluación de las capacidades de aprendizaje colaborativo adquiridas mediante el proyecto integrador de saberes
}

\author{
Ciaddy G. Rodríguez-Borges, Carlos A. Bowen-Quiroz, Jesús A. Pérez-Rodríguez y María Rodríguez-Gámez \\ Facultad de Ciencias Matemáticas, Físicas y Químicas, Universidad Técnica de Manabí, Av. Urbina y Che Guevara, \\ Portoviejo, Ecuador (correo-e: rodriguezciaddy@gmail.com)
}

Recibido Mar. 31, 2020; Aceptado Jun. 2, 2020; Versión final Jul. 15, 2020, Publicado Dic. 2020

\begin{abstract}
Resumen
Esta investigación consistió en evaluar el desarrollo de las capacidades de aprendizaje colaborativo adquiridas con el proyecto integrador de saberes y en evaluar la percepción de los estudiantes de ingeniería industrial de la Universidad Técnica de Manabí (Ecuador). Se trata de una investigación aplicada, cuantitativa y exploratoria, que siguió una metodología de investigación experimental. El diseño metodológico consistió en clasificar a la carrera en tres niveles y analizar, mediante un instrumento, las capacidades de aprendizaje colaborativo de los estudiantes y su percepción ante este proceso. Se obtuvo como resultado que existe mejora en las capacidades de aprendizaje colaborativo en el transcurso de la carrera, siendo la responsabilidad individual la capacidad más desarrollada. Además, se determinó que en todos los niveles existe, generalmente, una percepción positiva del proceso. Se concluye que hay una necesidad de que el proyecto integrador de saberes haga énfasis en fomentar las capacidades de aprendizaje colaborativo menos desarrolladas.
\end{abstract}

Palabras clave: aprendizaje cooperativo; aprendizaje activo; ingeniería industrial; percepción, innovación educativa

\section{Assessment of collaborative learning skills acquired through the knowledge integration project}

\begin{abstract}
The present research study assesses the collaborative learning capacities acquired with the knowledge integration project and the perception of industrial engineering students towards this methodology at the Technical University of Manabí (Ecuador). This is an applied quantitative exploratory research study that applied an experimental research methodology. The industrial engineering degree is classified in three levels and student perception and collaborative learning capacities are analyzed. The results show improvement in student collaborative learning capacities during the degree study. Individual responsibility is the most developed capacity and there is an overall positive student perception of the process. It is concluded that the knowledge integration project should emphasize the promotion of the least developed collaborative learning capacities.
\end{abstract}

Keywords: collaborative learning; active learning; industrial engineering; perception, educational innovation 


\section{INTRODUCCIÓN}

Las instituciones de educación superior de diversos países, entre ellos: Colombia, Venezuela, México y Ecuador, han venido implementando desde hace más de una década una estrategia metodológica en sus planes curriculares, denominada Proyecto Integrador de Saberes (PIS) (Acosta et al., 2017; Hewitt y Barreno, 2012; Materan, 2017; Suárez et al., 2018; Rivero et al., 2017). Esta estrategia persigue el objetivo de sistematizar y evidenciar los conocimientos alcanzados en cada nivel de la carrera, a través de un proyecto dirigido a la solución de un problema real, en el cual los estudiantes deben aplicar los conocimientos adquiridos y los recursos disponibles en su comunidad (Rodríguez-Torres et al., 2018). El PIS se empezó aplicar de forma obligatoria en el año 2013 en todas las universidades de Ecuador (Consejo de Educación Superior, 2013), incluida la UTM, por lo que se encuentra incorporado en los rediseños curriculares de todas las carreras desde la fecha señalada. Los contenidos de cada semestre se evalúan e integran para alcanzar un constructo de aprendizaje cuyo hilo conductor sea el PIS.

EI PIS como metodología está basado en la búsqueda de estrategias y recursos para llevar a la práctica los contenidos teóricos impartidos en el aula, a través de un proyecto de investigación interdisciplinario que permita la integración de saberes y habilidades de la disciplina de la carrera (Acosta et al., 2017). El actual contexto económico y social requiere profesionales con habilidades socio-emocionales (Estrada et al., 2016) para el trabajo en equipos multidisciplinarios. Dada esta demanda, el PIS como metodología se vuelve idóneo, pues los estudiantes deben proceder a investigar, plantearse hipótesis y posteriormente dar solución a un problema definido, desde un rol de innovadores y emprendedores, desde el cual son capaces de aplicar conocimientos para transformar y crear, apoyados en la institución de la cual forman parte, facilitando el desarrollo de habilidades profesionales y de trabajo colaborativo (Pérez et al., 2020). La implementación de esta estrategia requiere un cambio metodológico del rol del docente, donde además de ser un ente motivador, debe potenciar nuevas habilidades en los estudiantes, como por ejemplo, la capacidad de aprendizaje autónomo; además, el docente debe procurar actuar como tutor del aprendizaje, e ir direccionando en todo momento a los estudiantes hacia el desarrollo de habilidades cognitivas, comunicativas y de trabajo colaborativo, planteándose un cambio en la forma de entender el rol del estudiante en el proceso de enseñanza-aprendizaje (Pérez et al., 2020; Rodríguez et al., 2020; Zhou, 2017).

Antes de profundizar en la concepción del término aprendizaje colaborativo, es importante señalar que existen dos términos con acepciones similares, los cuales son: aprendizaje cooperativo y aprendizaje colaborativo. Diversos autores señalan diferencias, y otros tantos afirman que presentan similitudes e incluso emplean ambos términos de forma intercambiable (Andreu-Andrés, 2015). Por lo antes señalado, fue necesario revisar las diferencias señaladas, para identificar con precisión el término a emplear. En cuanto al aprendizaje cooperativo, se señala que puede existir heterogeneidad y diversidad entre los miembros del grupo, siendo el docente quien dirige la estructuración del proceso y la asignación de tareas a cada uno de los integrantes, que luego se organizarán para unificar los resultados alcanzados (Johnson y Johnson, 2014; Slavin, 2014).

Entre los autores que señalan similitudes entre el aprendizaje cooperativo y el colaborativo, se encuentra Crook (2013), quien plantea que solo pueden definirse ligeras diferencias, como el caso del énfasis dado al proceso cognitivo en el proceso colaborativo, más que lo motivacional. Se señalan, además, la existencia de similitudes y complementariedades entre ambos tipos de aprendizaje, ya que son la consecuencia de la integración del aporte de las teorías de Jean Piaget y Lev Vygotsky, por lo que estos aprendizajes están basados en el constructivismo, que fomenta la interacción social, el intercambio de ideas y conocimiento entre los miembros de un grupo (Grant, 2016).

En otros análisis sobre las similitudes (Pinho-Lopes y Macedo, 2016), se concluye que es posible emplear los términos aprendizaje cooperativo y colaborativo como símiles, entendiendo que el colaborativo, ha sido definido de forma reciente, como el aprendizaje que aborda las estrategias de enseñanza cooperativas a la vez otorga un mayor énfasis a las exigencias de: coordinación entre los miembros del grupo, aprendizaje autónomo y proceso cognitivo. Por lo que se entenderá, a los efectos de esta investigación, que el aprendizaje colaborativo, es aquel busca la elaboración de un constructo de aprendizaje, mediante la interacción, intercambio de ideas y conocimientos entre los miembros de un grupo, que ejecutan una tarea cuyos objetivos no solo comprenden la realización de la misma, sino también el desarrollo de habilidades individuales y grupales mediante el intercambio de roles de los miembros del grupo.

El aprendizaje colaborativo permite el desarrollo de cinco competencias fundamentales, para las cuales existe consenso entre diferentes autores (Damon, 1984; Gennari et al., 2016; Johnson y Johnson, 2014; León et al., 2017; Slavin, 2014). Estas son: a) independencia positiva, en la que los integrantes del grupo dependen de sus interacciones para alcanzar el objetivo, desarrollando cohesión social en el proceso; b) interacción promotora, que es el aspecto motivacional que se genera en los estudiantes cuando trabajan en grupo; c) responsabilidad individual, que permite que cada integrante asuma una parte del trabajo; d) procesamiento 
grupal, que permite la interacción con diferente niveles de desarrollo cognitivo, estimulando la creación de capacidades de aprendizaje y; e) habilidades sociales, que contempla las habilidades de comunicación interpersonal, liderazgo, gestión de tareas, entre otras. En consecuencia, cada uno de los elementos anteriores, resultan desarrollados durante un proceso satisfactorio de aprendizaje colaborativo.

Para la evaluación del aprendizaje colaborativo, se han realizado diferentes tipos de cuestionarios, que permiten medir las competencias fundamentales de esta estrategia. Pero, por ejemplo, Axturra et al. (2015) no consideran la responsabilidad individual como competencia del aprendizaje colaborativo y plantean que la valoración del aprendizaje se realice mediante observadores externos y no los propios participantes. Después de una amplia revisión bibliografía, se procedió a seleccionar un cuestionario que abordara las cinco competencias, cuya valoración fuese realizada por los propios participantes y que estuviese validado, seleccionándose uno en el que los autores, permiten estimar individualmente cada competencia y determinar un factor de cooperación global, lo que a su vez permite medir el desempeño individual y general de las capacidades de aprendizaje colaborativo

El aprendizaje colaborativo, no solo abarca el desarrollo de las competencias en los estudiantes (que serán medidas mediante la aplicación del cuestionario seleccionado), sino que actúa en el campo de las percepciones respecto a este tipo de aprendizaje, influenciado por las experiencias previas del estudiante, que lo impulsará a un mayor involucramiento en las tareas y roles a desempeñar (continuará desarrollando las competencias) o por el contrario, percibirá como no satisfactorio la aplicación de esta estrategia y tendrá una mayor tendencia al trabajo individual. Por ello, se consideró relevante aplicar un cuestionario complementario que permita identificar las ventajas y desventajas (percepciones del estudiante) del proceso de aprendizaje colaborativo.

En la Fig. 1, se ilustra el ciclo de percepción y desarrollo de las competencias del aprendizaje colaborativo, como punto de inicio se tiene la percepción de las experiencias previas, que influye considerablemente en el desarrollo de la percepción de la experiencia actual. Una vez asignado el trabajo, intervienen las primeras etapas de involucramiento con los miembros del grupo y la adopción de roles, que implícitamente abarcan la competencia de responsabilidad individual. Es desde estas etapas que el estudiante va consolidando su percepción hacia el proceso, basado en la comodidad con los miembros del grupo y el rol que adopta según las habilidades personales que demuestre. Si el estudiante no se siente cómodo con su equipo y con el rol que adoptó, tenderá a la responsabilidad individual, enfocándose en cumplir con sus tareas asignadas y culminar el proceso lo más pronto posible, evitando la fase de interacción para el intercambio de ideas y conocimientos, que es la etapa donde el estudiante desarrolla las cuatro competencias restantes del aprendizaje colaborativo. Si se omite esta etapa, el desarrollo cognitivo se ve interrumpido, aunque se haya alcanzado el objetivo planteado. De esta forma, el estudiante no valora las ventajas del aprendizaje colaborativo y su percepción del proceso tiende a la negatividad. Finalmente, la experiencia vivida retroalimenta la percepción general hacia el aprendizaje colaborativo para futuras experiencias.

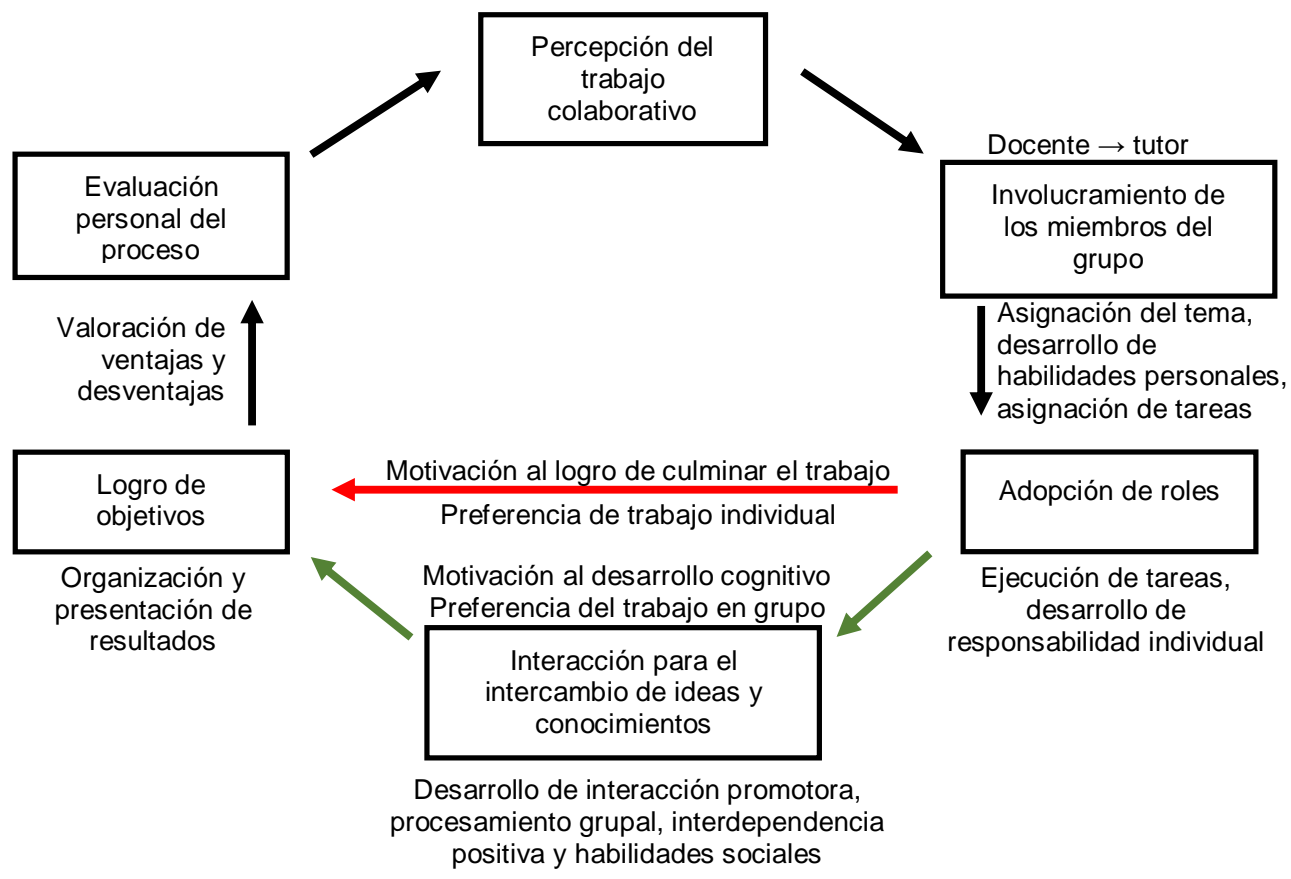

Fig. 1: Ciclo de percepción y desarrollo de capacidades de aprendizaje colaborativo 
Esta investigación parte de las hipótesis de que las experiencias iterativas de realizar los PIS, en cada uno de los niveles a lo largo de la carrera, permiten desarrollar las capacidades de aprendizaje colaborativo y la percepción de los estudiantes sobre este proceso de aprendizaje, ambos aspectos deben evaluarse para alcanzar mejoras en la ejecución de la estrategia. El objetivo es efectuar un diagnóstico de la evolución de las capacidades de aprendizaje colaborativo y la percepción de los estudiantes sobre el proceso de aprendizaje durante el desarrollo del PIS, en el transcurso de la carrera de ingeniería industrial de la Universidad Técnica de Manabí (UTM), Ecuador. Este estudio es relevante en el contexto ecuatoriano, dada la necesidad de corroborar el desenvolvimiento de esta estrategia, que se encuentra normada en el Reglamento de Régimen Académico del Consejo de Educación Superior (2013) como obligatoria para el desarrollo de todos los planes curriculares de las diferentes carreras universitarias (Quiñonez, 2018). Se analizará de forma particular una carrera técnica, como es el caso de ingeniería industrial, en la que se amerita desarrollar en el estudiantado competencias para el trabajo en grupo (Suárez et al., 2018).

\section{METODOLOGÍA}

Se trata de una investigación aplicada, cuantitativa y exploratoria, que siguió una metodología de investigación experimental, en la cual el medio de obtención de datos fue la encuesta, que fue aplicada a los estudiantes de la carrera de ingeniería industrial de la UTM, en Ecuador, a través de los docentes que coordinan el PIS en cada semestre de la carrera, por medio de los grupos de contacto. La encuesta se realizó a través de la plataforma Formularios de Google, durante la última semana de enero del 2020. Los niveles se clasificaron de acuerdo al avance académico, obteniéndose los siguientes segmentos para este análisis: nivel entrante (de 1 a 3 semestre), nivel intermedio (de 4 a 6 semestre) y nivel saliente (de 7 a 9 semestre). Se consideró irrelevante la adición de la variable género en la encuesta, debido a que el estudio se enfocó en medir la evolución de las capacidades de los estudiantes por nivel de carrera y no en hacer una diferenciación de las capacidades por género.

Se calculó el tamaño de la muestra correspondiente al total de estudiantes de la carrera, considerando que la población es de 398, 261 y 130 en los niveles entrante, intermedio y saliente respectivamente. El margen de confianza fijado fue de $95 \%$ y el margen de error fue de 5\%; por lo que al calcular la debida muestra (Gupta et al., 2016), los resultados fueron los siguientes: 196, 156 y 98 estudiantes a encuestar para los niveles entrante, intermedio y saliente respectivamente.

\section{Cuestionario de Aprendizaje Cooperativo (CAC)}

La primera parte de la encuesta consistió en un cuestionario desarrollado por Fernández-Rio et al. (2017), docentes de la Facultad de Formación del Profesorado y Educación de la Universidad de Oviedo, España, bajo la premisa de que los instrumentos creados hasta la fecha no contemplan todos los componentes fundamentales del aprendizaje colaborativo. Se escogió este instrumento ya que fue estadísticamente validado, cuenta con buenas propiedades psicométricas, evalúa los 5 componentes del aprendizaje cooperativo y es de corta extensión en cuanto al número de preguntas. El CAC está formado por 20 enunciados, que deben ser evaluados por el estudiante de acuerdo con el grado de acuerdo o desacuerdo que percibe de estos, basándose en la escala de Likert (Joshi et al., 2015), que asigna una escala numérica tal que 1 representa el total desacuerdo y 5 el total acuerdo. Los enunciados están divididos en 5 grupos (4 enunciados por grupo), que califican cada componente del aprendizaje cooperativo por separado y en su conjunto, el nivel de aprendizaje cooperativo en general. En el presente trabajo se concuerda con los autores del CAC, quienes distribuyeron los enunciados de tal forma que el encuestado no descifre la variable que se intenta obtener de él. Se realizaron adaptaciones ligeras de los enunciados en cuestiones de sinonimia de términos, más no en el contexto de los mismos, con el fin de que el instrumento se adapte a su entorno de aplicación (un país y sociedad diferente). Al tratarse de modificaciones de forma, mas no de fondo, no se altera el instrumento, no se requiere revalidación por expertos y los resultados obtenidos siguen siendo válidos.

La calificación por componente se obtiene al sumar los valores de la respuesta de cada enunciado, de forma que, estar totalmente de acuerdo con todos los enunciados equivale al nivel máximo de aprendizaje colaborativo (100 puntos). El factor de cooperación global proviene de la suma de cada componente (20 puntos por componente como máximo). Se definieron tres niveles de aprendizaje colaborativo: alto, medio y bajo, basado en la clasificación realizada por Mejía (2019). Si bien se adoptó la terminología del nivel de aprendizaje colaborativo de este trabajo, no se estuvo de acuerdo con los rangos allí planteados, por lo que se formularon nuevos rangos descritos a continuación. Se consideró que el mínimo aceptable para obtener un nivel alto es 16, que equivale a estar por lo menos "de acuerdo" en todos los enunciados de una categoría. Por otra parte, la obtención de un puntaje igual o menor a 12, lo cual corresponde a "no opinar" en todos los enunciados o a no estar de acuerdo en ninguno de los enunciados, dando como resultado un nivel bajo. Por último, el rango restante (nivel medio), que se encuentra entre 13 y 15 , equivale a estar por lo menos "de 
acuerdo" en un enunciado. Cada uno de los componentes del aprendizaje colaborativo se compararon entre sí y entre niveles, con el fin de evaluar la evolución de las competencias en el transcurso de la carrera.

\section{Percepción del alumnado ante el aprendizaje colaborativo}

La segunda parte de la encuesta evaluó la percepción de los estudiantes respecto a las ventajas y desventajas que presenta el aprendizaje colaborativo, señalando las preferencias implícitas de los estudiantes hacia la metodología tradicional o la de aprendizaje colaborativo. Para esto se empleó un conjunto de opciones que representaban las ventajas y desventajas de este tipo de aprendizaje, previamente planteadas por Escolano et al. (2012). Para no condicionar al estudiante a escoger ventajas o desventajas que no reconoce, se antepuso una pregunta de ramificación condicional, de forma que únicamente los estudiantes que reconocen la existencia de estos aspectos pudiesen seleccionarlos. De igual forma, se permitió al estudiante escoger una, dos o tres ventajas o desventajas como máximo, si así lo consideraba, de forma que su percepción no se viera limitada.

\section{RESULTADOS Y DISCUSIÓN}

En la carrera de ingeniería industrial de la UTM existe la necesidad de evaluar las capacidades de aprendizaje colaborativo adquiridas a lo largo de la carrera a través del PIS, para corroborar el desenvolvimiento de esta estrategia en la formación profesional de los estudiantes, los cuales requieren en el desenvolvimiento de su ejercicio profesional la continua integración a equipos multidisciplinarios, y por ende, de capacidades de trabajo colaborativo (Suárez et al., 2018). El desarrollo del PIS generalmente se realiza en dos etapas, una primera de revisión teórica, donde se aportan todos los conceptos de las materias a integrar, en conjunto con la metodología para realizarlo. Se debate el "objeto" donde será aplicado el conocimiento (instalación, prototipo, artefactos, entre otros). Se conforman los grupos de trabajo, y se expone la metodología de trabajo colaborativo y los elementos que componen el aprendizaje (que serán evaluados). Tras conocer la temática del proyecto, los grupos de trabajo plantean los objetivos y se utiliza la dinámica de tormenta de ideas para seleccionar el "objeto" donde se realizará el PIS. En la segunda etapa se imparten sesiones guiadas para el desarrollo de las prácticas y de la solución del caso de estudio. Finalmente, de forma grupal, se presentan los resultados del proyecto, como trabajo de fin de curso, donde no solo se evalúa el resultado, sino también el proceso para alcanzarlo. Fue en la fase final de PIS donde se procedió a realizar la encuesta, de acuerdo a la muestra estimada por cada nivel. Dados los resultados, una vez aplicada la encuesta, se realizó una revisión a las respuestas aportadas, encontrándose la existencia de respuestas con data viciada (tendencia constante a valorar los enunciados de forma idéntica a lo largo de todo el cuestionario). Debido a esto, fue necesario realizar un proceso de filtrado de las respuestas obtenidas, procediendo a eliminar la encuesta en su totalidad, para aquellos casos que demuestren las consideraciones antes señaladas. Se obtuvo un total de encuestas válidas de 134, 116 y 53 para los niveles entrante, intermedio y saliente respectivamente.

\section{Cuestionario de Aprendizaje Cooperativo (CAC)}

Un aspecto importante evidenciado en la aplicación del CAC es la ausencia de alternancia en el sentido positivo de los enunciados, lo que puede estimular al estudiante a completar la encuesta de forma automática, es decir, sin lectura crítica, dado que es factible predecir que en la medida que su percepción corresponda a la máxima (totalmente de acuerdo) se entenderá que su puntuación en el manejo de la metodología es la mejor. Esto se consideró una deficiencia del instrumento, dado el número de encuestas completadas sin criterio (147). En la Tabla 1, se muestra la valoración cuantitativa y cualitativa de los componentes del aprendizaje colaborativo, así como la valoración general por cada nivel de la carrera.

Tabla 1. Valoración cualitativa y cuantitativa de las capacidades de aprendizaje colaborativo

\begin{tabular}{|l|l|l|l|l|l|l|}
\hline \multirow{2}{*}{$\begin{array}{l}\text { Componente del aprendizaje } \\
\text { colaborativo }\end{array}$} & \multicolumn{4}{l}{ Valoración cuantitativa } & \multicolumn{3}{l|}{ Valoración cualitativa } \\
\cline { 2 - 8 } & Entrante & Intermedio & Saliente & Entrante & Intermedio & Saliente \\
\hline Habilidades sociales & 13 & 14 & 15 & Medio & Medio & Medio \\
\hline Procesamiento grupal & 13 & 14 & 15 & Medio & Medio & Medio \\
\hline Interdependencia positiva & 13 & 14 & 15 & Medio & Medio & Medio \\
\hline Interacción promotora & 13 & 14 & 15 & Medio & Medio & Medio \\
\hline Responsabilidad individual & 14 & 15 & 16 & Medio & Medio & Alto \\
\hline Factor de cooperación global & 66 & 71 & 75 & Medio & Medio & Medio \\
\hline
\end{tabular}


Valorando cuantitativamente los resultados es posible observar que existe una mejora en la valoración de las capacidades de aprendizaje colaborativo en cada nivel. No se observa una diferencia significativa entre las capacidades de aprendizaje colaborativo de un nivel en particular de la carrera, todos satisfactoriamente avanzan a medida que se van realizando los procesos del PIS; $y$ en todos los casos existe un aumento en el puntaje entre niveles, conforme se avanza en la carrera. El componente de aprendizaje colaborativo mejor valorado es el de responsabilidad individual, quizás explicado por la cultura individualista predominante en esquemas de aprendizaje anteriores al universitario. Sin embargo, el aumento de esta capacidad entre niveles fue el mismo que para las demás capacidades. Esta capacidad destacada hace referencia a la habilidad de cada individuo del grupo para cumplir con su rol. Esto difiere de lo obtenido por Mejía (2019), quien aplicó el CAC y halló que la capacidad más desarrollada del grupo estudiado que fue la de habilidades sociales, mientras que la menos desarrollada fue la responsabilidad individual. En general, existe una evolución de las capacidades de aprendizaje colaborativo en los estudiantes de ingeniería industrial.

\section{Percepción del alumnado ante el aprendizaje colaborativo}

Respecto a la percepción de ventajas y desventajas por parte del estudiante, representadas en la Fig. 2, se observa que, en el nivel entrante, el porcentaje que percibe ventajas es mayor en comparación a los niveles restantes, haciendo énfasis en el nivel intermedio, donde la percepción de ventajas fue mínima (81\%). Por otra parte, el número de estudiantes que perciben desventajas tuvo un crecimiento en cada nivel, donde el menor porcentaje se registró en el nivel entrante (37\%), incrementándose a un $42 \%$ en el nivel intermedio y a un $45 \%$ de porcentaje de percepción de desventajas en el nivel saliente. La tendencia de aumento de percepción de desventajas es notoria en el transcurso de los niveles de la carrera. Las figuras 3 y 4 que explican la frecuencia con la que los estudiantes perciben las ventajas y las desventajas planteadas, respectivamente. Los resultados de cada nivel de la carrera se comparan entre sí.

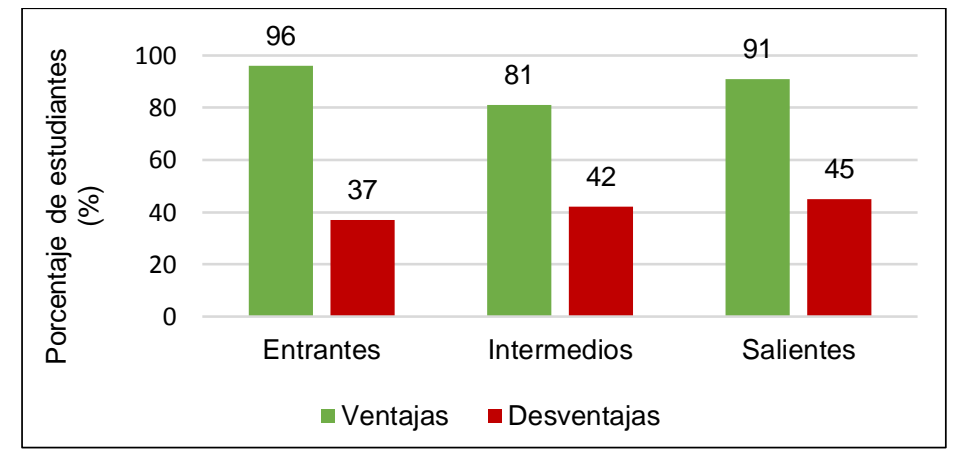

Fig. 2: Porcentaje de estudiantes que perciben ventajas y desventajas.

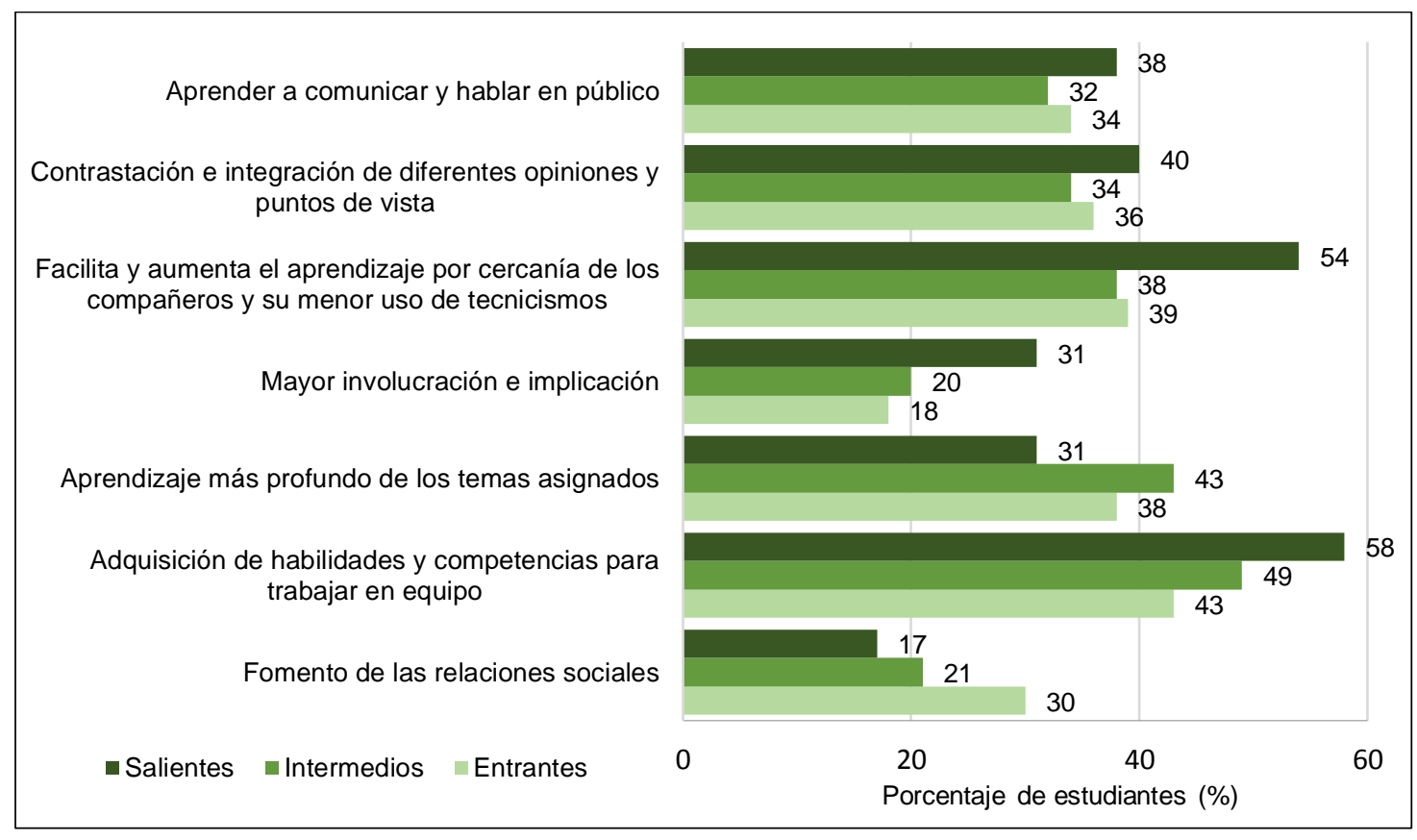

Fig. 3: Frecuencia de percepción de ventajas por nivel 
Como se muestra en la Fig. 3, las ventajas percibidas con mayor frecuencia en todos los niveles de la carrera fueron las de adquisición de habilidades y competencias para trabajar en equipo, la de facilidades y aumento del aprendizaje por cercanía con los compañeros y menor uso de tecnicismos. El nivel saliente percibe en mayor medida las ventajas antes señaladas, lo que corrobora que existe un refuerzo positivo de la percepción del trabajo en grupo. No obstante, no todos los estudiantes percibieron ventajas, y otros identificaron únicamente una o dos. Las ventajas menos percibidas fueron las de fomento de las relaciones sociales y mayor involucración e implicación. Esto supone que este tipo de metodologías colaborativas tienen poca incidencia en las relaciones sociales de los estudiantes.

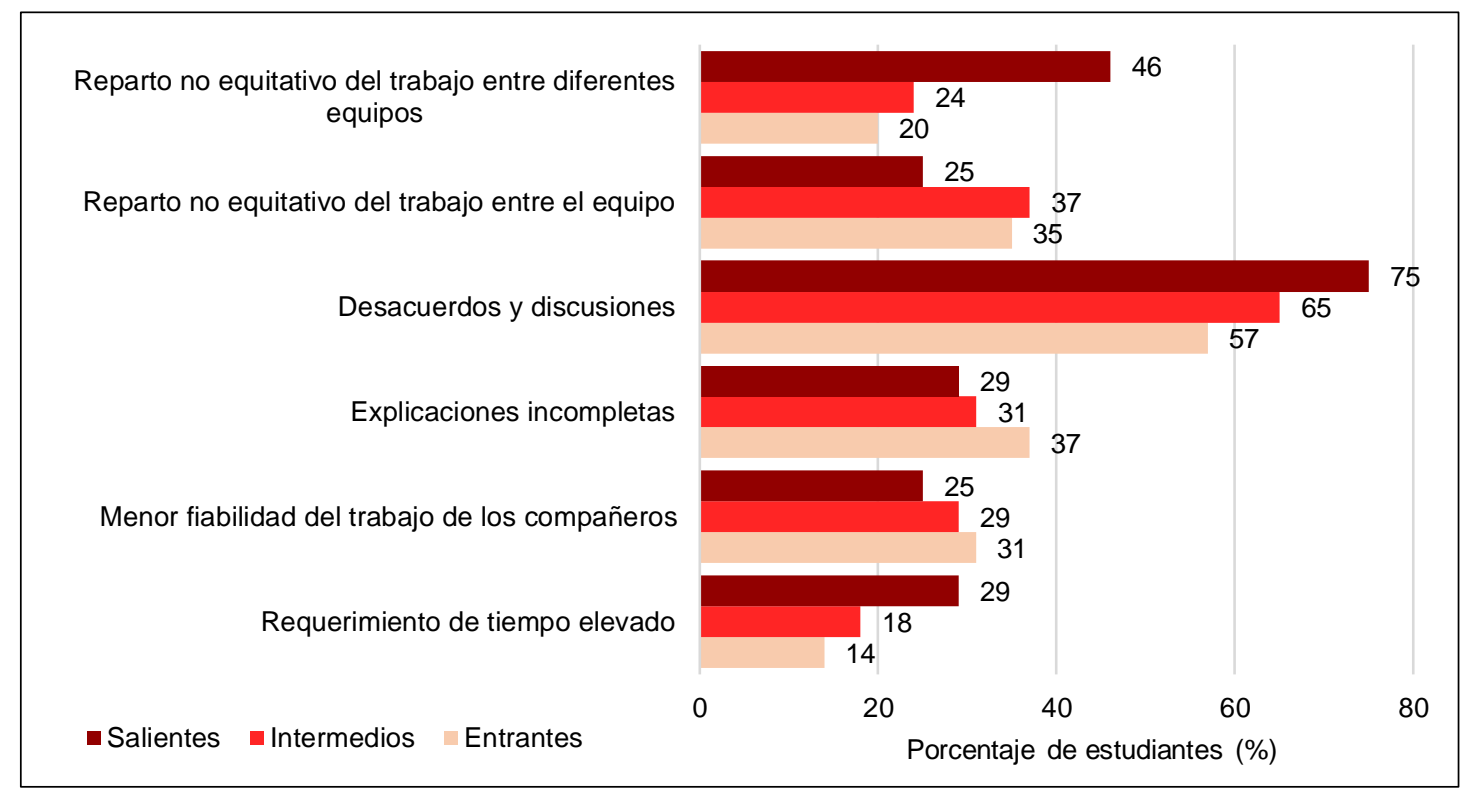

Fig. 4: Frecuencia de percepción de desventajas por nivel.

En la Fig. 4, que describe las desventajas, se observa un comportamiento similar en cuanto a que el nivel saliente es quien más percibe desventajas, siendo la de mayor frecuencia la de desacuerdos y discusiones, la cual predomina como la más percibida por los niveles restantes. Esto puede guardar cierta relación con el comportamiento del estudiante de omitir la fase de interacción para el intercambio de ideas y conocimientos, pues se genera una percepción negativa de la metodología.

\section{CONCLUSIONES}

Sobre las capacidades de aprendizaje colaborativo de los estudiantes de ingeniería industrial de la UTM, adquiridas mediante la práctica iterativa y sistemática del PIS a lo largo de la carrera, se puede concluir que:

1) Existe una ligera evolución de las capacidades de aprendizaje colaborativo, especialmente la capacidad de responsabilidad individual.

2) El menor desarrollo de las capacidades diferentes a la responsabilidad individual puede estar relacionado a la omisión de la fase de interacción para el intercambio de ideas y conocimientos, que a su vez puede partir de la percepción negativa que los estudiantes tienen del proceso.

3) La implementación del PIS y su metodología deben ser revisadas, con el fin de alcanzar un mayor desarrollo de las capacidades de aprendizaje colaborativo.

4) Se deben contemplar acciones para incentivar al estudiante a involucrarse activamente en la fase de interacción para el intercambio de ideas y conocimientos, a comprender la importancia de esta fase para su desarrollo cognitivo y a mantener la armonía con los miembros del grupo. Aplicar estrategias didácticas como el aprendizaje basado en problemas y el estudio de casos en el marco de trabajo del proyecto integrador pueden incentivar al estudiante a involucrarse en la fase interactiva.

\section{REFERENCIAS}

Acosta, M.L., Ocampo, C.H., y Corona, M.S., Proyectos integradores. Una estrategia para desarrollar proyectos sustentables en el aula, https://doi.org/10.19136/hitos.a0n67.2190, Hitos de Ciencias Económico Administrativas, (67), 343-359 (2017).

Andreu-Andrés, M.A., Cooperative or collaborative learning: is there a difference in university students' perceptions?, https://doi.org/10.5209/rev_RCED.2016.v27.n3.47398, Revista Complutense de Educación, 27(3), 1041-1060 (2015). 
Axturra, C., Villardón-Gallego, L. y Calvete, E., Diseño y Validación de la Escala de Aplicación del Aprendizaje Cooperativo (CLAS), https://doi.org/10.1387/RevPsicodidact.11917, Revista de Psicodidáctica, 20(2), 339-357 (2015).

Consejo de Educación Superior, Reglamento de Régimen Académico. Gaceta oficial ecuatoriana (2013).

Crook, C., Varieties of "togetherness" in learning-and their mediation affective learning together, New perspectives on Learning and Instruction, 33-51 (2013).

Damon, W., Peer education: the untapped potential, https://doi.org/10.1016/0193-3973(84)90006-6, Journal of Applied Developmental Psychology, 5(4), 331-343 (1984).

Escolano, E., Tomás, L. y Herrero, M.L., Percepción del alumnado universitario sobre su primera experiencia de aprendizaje colaborativo, V Congreso Mundial de Estilos de Aprendizaje, Santander (2012).

Estrada, M., Monferrer, D. y Moliner, M.A., El aprendizaje cooperativo y las habilidades socio-emocionales: una experiencia docente en la asignatura técnicas de ventas, https://doi.org/10.4067/S0718-50062016000600005. Formación Universitaria, 9(6), 43-62 (2016).

Fernández-Rio, J., Cecchini, J. A. y otros 3 autores, Design and validation of a questionnaire to assess cooperative learning in educational contexts, https://doi.org/10.6018/analesps.33.3.251321, Anales de Psicologia/Annals of Psychology, 33(3), 680-688 (2017).

Gennari, R., Melonio, A. y Torello, S., Gamified probes for cooperative learning: a case study, https://doi.org/10.1007/s11042-016-3543-7, Multimedia Tools and Applications, 76(4), 4925-4949 (2016).

Grant, C.B., Constructivism, https://doi.org/10.1002/9781118766804.wbiect160, The International Encyclopedia of Communication Theory and Philosophy, 1-9 (2016).

Gupta, K.K., Attri, J.P. y otros 3 autores, Basic concepts for sample size calculation: critical step for any clinical trials!, https://doi.org/10.4103/1658-354X.174918, Saudi Journal of Anaesthesia, 10(3), 328-331 (2016).

Hewitt, N. y Barrero, F., La integración de los saberes: una propuesta curricular para la formación en investigación en la educación superior, Psychologia, 6(1), 137-145 (2012).

Johnson, D.W. y Johnson, R.T., Cooperative learning in 21st century, https://doi.org/10.6018/analesps.30.3.201241, Anales de Psicología / Annals of Psychology, 30(3), 841-851 (2014).

Joshi, A., Saket, K. y otros 2 autores, Lickert scale: explored and explained, https://doi.org/10.9734/BJAST/2015/14975, British Journal of Applied Science \& Technology, 7(4), 396-403 (2015).

León, B., Mendo-Lázaro, S. y otros 3 autores, Team potency and cooperative learning in the university setting, https://doi.org/10.1387/RevPsicodidact.14213, Revista de Psicodidáctica (English Edition), 22(1), 9-15 (2017).

Materan, C., Corresponsabilidad tríadica en el desarrollo del proyecto socio integrador, Daena: International Journal of Good Conscience, 12(3), 141-156 (2017).

Mejía, C.A., Aprendizaje Cooperativo y Habilidades Sociales en Estudiantes de Medicina de una Universidad de Lima (Tesis de maestría). Universidad César Vallejo, Lima, Perú (2019).

Pérez, J., Rodríguez, C. y otros 2 autores, Emulation of systems as strategy for teaching of mechanical systems, https://doi.org/10.37200/IJPR/V24I2/PR200368, International Journal of Psychosocial Rehabilitation, 24(2), 544-550 (2020).

Pérez, J.A., Rodríguez, C.G. y otros 2 autores, Espacios maker: herramienta motivacional para estudiantes de ingeniería eléctrica de la Universidad Técnica de Manabí, Ecuador. Espacios, 41(2), 12p (2020).

Pinho-Lopes, M. y Macedo, J., Project-based learning in geothecnics: cooperative versus collaborative teamwork, https://doi.org/10.1080/03043797.2015.1056099, European Journal of Engineering Education, 41(1), 70-90 (2015).

Quiñonez, E.S., Proyecto integrador de saberes, evidencia del resultado de aprendizaje. INNOVA Research Journal, 3(3), 84-94 (2018).

Rivero, M., Campuzano, G.P. y Ferrer-Sánchez, Y., Proyecto integrador: una herramienta metodológica en la educación superior. Revista Didasc@lia: Didáctica y Educación, 8(1), 241-250 (2017).

Rodríguez-Torres, Á.F., Fierro-Altamirano, R.H. y otros 2 autores, La resolución de problemas: una oportunidad para aprender a aprender (original). Revista científica OLIMPIA, 15(50), 160-171 (2018).

Rodríguez, C., Pérez, J. y otros 3 autores, Software development for transformer model supporting significant learning electrical machines, https://doi.org/10.37200/IJPR/V24I2/PR200373, International Journal of Psychosocial Rehabilitation, 24(2), 591-599 (2020).

Slavin, R.E., Cooperative learning and academic achievement: why does groupwork work?, https://doi.org/10.6018/analesps.30.3.201201, Anales De Psicología / Annals of Psychology, 30(3), 785-791 (2014).

Suárez, N., Martínez, A. y Lara, D.G., Interdisciplinariedad y proyectos integradores: un desafío para la universidad ecuatoriana, https://doi.org/10.4151/07189729-Vol.57-Iss.3-Art.700, Perspectiva Educacional, 57(3), 54-78 (2018).

Zhou, Z., On the strategy to enhance the efficiency of the cooperative learning method in the College English Teaching in China, https://doi.org/10.17507/tpls.0711.18, Theory and Practice in Language Studies, 7(11), 1091-1096 (2017). 